\title{
Symptomatic psychosis risk and physiological fluctuation in functional MRI data
}

\section{Saarinen, Aino}

2020-02

Saarinen , A , Lieslehto , J , Kiviniemi , V , Häkli , J , Tuovinen , T, Hintsanen , M \& Veijola , J 2020 , ' Symptomatic psychosis risk and physiological fluctuation in functional MRI data ' , Schizophrenia Research , vol. 216 , pp. 339-346 . https://doi.org/10.1016/j.schres.2019.11.029

http://hdl.handle.net/10138/322332

https://doi.org/10.1016/j.schres.2019.11.029

acceptedVersion

Downloaded from Helda, University of Helsinki institutional repository.

This is an electronic reprint of the original article.

This reprint may differ from the original in pagination and typographic detail.

Please cite the original version. 


\title{
Symptomatic psychosis risk and physiological fluctuation in functional MRI data
}

\author{
Aino Saarinen ${ }^{\mathrm{a}, \mathrm{b}, c, *, 1}$, Johannes Lieslehto ${ }^{\mathrm{c}, \mathrm{d}, 1}$, Vesa Kiviniemi ${ }^{\text {e,f }}$, Jani Häkli ${ }^{\mathrm{e}}$, Timo Tuovinen ${ }^{\mathrm{e}}$, \\ Mirka Hintsanen ${ }^{\mathrm{a}, 2}$, Juha Veijola ${ }^{\mathrm{c}, \mathrm{f}, \mathrm{g}, 2}$ \\ ${ }^{a}$ Research Unit of Psychology, University of Oulu, Finland \\ ${ }^{b}$ Department of Psychology and Logopedics, Faculty of Medicine, University of Helsinki, Finland \\ ${ }^{c}$ Research Unit of Clinical Neuroscience, Department of Psychiatry, University of Oulu, Finland \\ d Section for Neurodiagnostic Applications, Department of Psychiatry, Ludwig Maximilian University, Nussbaumstrasse 7, 80336 Munich, Bavaria, Germany \\ e Department of Diagnostic Radiology, Oulu University Hospital, Oulu, Finland \\ ${ }^{\mathrm{f}}$ Medical Research Center Oulu, Oulu University Hospital and University of Oulu, Oulu, Finland \\ ${ }^{g}$ Department of Psychiatry, Oulu University Hospital, Oulu, Finland
}

\section{A R T I C L E I N F O}

Article history:

Received 11 March 2019

Received in revised form 11 October 2019

Accepted 19 November 2019

Available online $\mathrm{xxxx}$

\section{Keywords:}

Prodromal symptoms

Psychosis, SIPS

fMRI

Physiological fluctuation

\begin{abstract}
A B S T R A C T
Background: Physiological brain pulsations have been shown to play a critical role in maintaining interstitial homeostasis in the glymphatic brain clearance mechanism. We investigated whether psychotic symptomatology is related to the physiological variation of the human brain using fMRI.

Methods: The participants $(N=277)$ were from the Northern Finland Birth Cohort 1986. Psychotic symptoms were evaluated with the Positive Symptoms Scale of the Structured Interview for Prodromal Syndromes (SIPS). We used the coefficient of variation of BOLD signal $\left(\mathrm{CV}_{\mathrm{BOLD}}\right)$ as a proxy for physiological brain pulsatility. The $\mathrm{CV}_{\mathrm{BOLD}}$-analyses were controlled for motion, age, sex, and educational level. The results were also compared with fMRI and voxel-based morphometry (VBM) meta-analyses of schizophrenia patients (data from the Brainmap database).

Results: At the global level, participants with psychotic-like symptoms had higher $\mathrm{CV}_{\mathrm{BOLD}}$ in cerebrospinal fluid (CSF) and white matter (WM), when compared to participants with no psychotic symptoms. Voxel-wise analyses revealed that $\mathrm{CV}_{\text {BOLD }}$ was increased, especially in periventricular white matter, basal ganglia, cerebellum and parts of the cortical structures. Those brain regions, which included alterations of physiological fluctuation in symptomatic psychosis risk, overlapped $<6 \%$ with the regions that were found to be affected in the metaanalyses of previous fMRI and VBM studies in schizophrenia patients. Motion did not vary as a function of SIPS. Conclusions: Psychotic-like symptoms were associated with elevated $\mathrm{CV}_{\mathrm{BOLD}}$ in a variety of brain regions. The $\mathrm{CV}_{\mathrm{BOLD}}$ findings may produce new information about cerebral physiological fluctuations that have been out of reach in previous fMRI and VBM studies.
\end{abstract}

(c) 2019 Elsevier B.V. All rights reserved.

\section{Introduction}

Traditionally, functional magnetic resonance imaging (fMRI) studies aim to investigate neuronal-activation-induced changes both spontaneous and task-related in the blood-oxygenation-level-dependent (BOLD) signal (Ogawa et al., 1990). Neuronal activation is coupled to a

* Corresponding author at: Department of Psychology and Logopedics, Faculty of Medicine, Haartmaninkatu 3, P.O. Box 21, 00014, University of Helsinki, Finland.

E-mail addresses: aino.i.saarinen@helsinki.fi (A. Saarinen),

johannes.pulkkinen@oulu.fi (J. Lieslehto), vesa.kiviniemi@oulu.fi (V. Kiviniemi), jani.hakli@student.oulu.fi (J. Häkli), timo.tuovinen@student.oulu.fi (T. Tuovinen), mirka.hintsanen@oulu.fi (M. Hintsanen), juha.veijola@oulu.fi (J. Veijola).

1 These authors contributed equally.

2 These authors contributed equally. hemodynamic response where $\mathrm{T} 2{ }^{*}$-weighted BOLD signal intensity increases after a hemodynamic delay of 3-5 s.

In addition to neuronal activity, there is also a set of other factors affecting the BOLD signal and physiological fluctuation in the brain. Such factors include cardiac and respiratory variations affecting cerebral blood volume and flow and oxygen and carbon dioxide extraction (Cheng et al., 2015; Krüger and Glover, 2001; Murphy et al., 2009). Physiological fluctuation may also be affected by cerebrospinal fluid flow (e.g. Mestre et al., 2018). The discovery of the glymphatic brain clearance mechanism by Nedergaard's group recently showed how the cardiovascular pulsations convey waste materials in the perivascular space in mice (Mestre et al., 2018). Along with this, there is evidence that also the human brain is pulsating markedly with cardiorespiratory frequencies and that these pulsations are altered in the areas 
controlling the respiration during breath-hold challenges (Kiviniemi et al., 2016; Raitamaa et al., 2018). Furthermore, cardiac and respiratory cycles are noted to induce changes in the cerebrospinal fluid flow into the conduits and ventricles (Birn et al., 2006; Birn, 2012; Weissenbacher et al., 2009; Dreha-Kulaczewski et al., 2015; Kiviniemi et al., 2016). Taken together, physiological fluctuation appears to derive from a variety of cardiorespiratory activities, cerebrospinal fluid flow, and the functioning of the glymphatic clearance system.

In most of the previous fMRI research, physiological fluctuation has been usually regarded as nuisance variation covering neuronalactivation related signal changes (Wise et al., 2004). Along with this, also fMRI studies in the field of psychotic disorders have made rigorous attempts to remove physiological signal sources from the fMRI data. Removing physiological fluctuation from the BOLD signal, however, may potentially exclude also valuable information about brain physiology. Previously, it has been demonstrated that physiological fluctuation contributes to a significant part, even 10\%, of the BOLD-signal variation (Birn et al., 2012; Dagli et al., 1999). Moreover, physiological fluctuations in the brain are not limited to the grey matter but are also found in the white matter and cerebrospinal fluid (Birn et al., 2006; Birn, 2012; Weissenbacher et al., 2009). Consequently, fMRI studies exploring differences in physiological fluctuation between various populations might provide novel insights into brain functioning. That is, it could provide the possibilities (i) to gain insights into the physiological activities in the brain (instead of merely neuronal activities), and (ii) to investigate neurophysiological functioning not only in grey matter but also in the white matter and cerebrospinal fluid.

Previous evidence suggests that changes in the physiological fluctuation of the brain tissues may potentially represent a biomarker for specific neurological or psychiatric disorders. For example, patients with Alzheimer's disease and small-vessel disease have increased physiological fluctuation in the white matter (Makedonov et al., 2013, 2016). Further, patients with epilepsy or acute ischemic stroke are found to have reduced physiological brain fluctuation (Kananen et al., 2018; Khalil et al., 2017; Wang et al., 2008). Regarding psychosis, however, the evidence is largely lacking. Until now, there exists only one study suggesting that schizophrenia might be related to increased physiological noise in the white matter, in the cerebellum and parietal lobes (Cheng et al., 2015). However, no study has investigated whether the changes in physiological brain fluctuation might be detected among individuals at symptomatic risk for developing psychosis, i.e. at the early stages of psychosis.

This study investigated whether psychosis risk is linked with BOLD signal fluctuations in the brain. We used data from the Oulu Brain and Mind study. All the participants were scanned with resting-state functional magnetic resonance imaging ( $r$-fMRI). We used the coefficient of variation $\left(\mathrm{CV}_{\mathrm{BOLD}}\right)$ as a proxy of physiological brain pulsations (Makedonov et al., 2013, 2016; Kananen et al., 2018; Khalil et al., 2017; Tuovinen et al., 2017). To identify those brain regions that have been robustly identified as schizophrenia-related, we conducted metaanalyses of fMRI and voxel-based morphometry (VBM) studies in patients with schizophrenia (data from the Brainmap database). We hypothesized that the association between $\mathrm{CV}_{\text {BOLD }}$ and SIPS would overlap with these meta-analytical maps.

\section{Material and methods}

\subsection{Participants}

The participants were selected from the Oulu Brain and Mind Study, which is a part of the Northern Finland Birth Cohort 1986 (NFBC 1986) study (Järvelin et al., 1997). The NFBC 1986 consists of individuals with an expected date of birth between July 1985 and June 1986 in the two northernmost provinces of Finland. The original sample of the NFBC 1986 included 9432 participants altogether.
The Oulu Brain and Mind Study was conducted in 2007-2010 for a subsample of the NFBC 1986. The aim of the Oulu Brain and Mind study was to investigate the developmental pathogenesis of psychosis among young people at risk for psychosis. The sample (total $N=329$ ) consisted of 5 groups: (i) participants with familial risk for psychosis, (ii) participants with symptomatic risk for psychosis, (iii) participants with previous psychosis, (iv) participants with attention-deficit/ hyperactivity disorder, and (v) healthy controls. Research-staff were not aware of the participants' invitation criteria. A more detailed description of the Oulu Brain and Mind Study and the NFBC 1986 are available elsewhere (Jukuri et al., 2013; Veijola et al., 2013).

The design of the NFBC 1986 study and the Oulu Brain and Mind Study were approved by the Ethics Committee of the Northern Ostrobothnia Hospital District in Finland. The studies were carried out in accordance with the Declaration of Helsinki. All the participants provided written informed consent after the nature of the procedures was fully explained.

In the present study, we excluded all the participants with a positive urine drug test for opiates, benzodiazepines, and cannabis $(N=22)$; participants with current use of benzodiazepines, neuroleptics, or other psychiatric medication $(N=17)$; inadequate or missing brain scan data $(N=12)$; or missing data about educational level $(N=1)$. The final sample consisted of 277 participants.

\subsection{Measures}

\subsubsection{Psychotic symptomatology}

Psychotic symptomatology was evaluated with the Structured Interview for Prodromal Syndromes (SIPS, version 3.0) (McGlashan et al., 2001). The SIPS measures three separate prodromal syndromes: brief intermittent psychotic syndrome, attenuated positive prodromal syndrome, and genetic risk and deterioration syndrome. The reliability and validity of the SIPS have been confirmed previously (Miller et al., 2003). In the present study, each participants' psychotic symptomatology was defined as the highest score of the SIPS positive symptoms within the past month. The score of the SIPS positive symptoms ranged from 0 (absence of psychotic symptoms) to 6 (psychotic symptoms). This score was further recoded into 4 categories as follows: (1) the score of 0 (no psychotic symptoms); (2) the scores of 1-2 (mild psychotic-like symptoms); (3) the scores of 3-5 (prodromal symptoms of psychosis); (4) the score of 6 (psychotic symptoms).

\subsubsection{Participants' background characteristics}

Background information was collected about participants' age, sex, educational level, smoking status, alcohol use, current Axis-I disorders, presence of neurological disorders, times of having been unconscious, level of functioning, and full-scale intelligence quotient.

Educational level was assessed with a self-report questionnaire. Educational level was classified into 2 categories $(1=$ comprehensive school or less; 2 = matriculation).

Smoking status was evaluated by asking participants whether they had ever smoked cigarettes regularly $(1=$ no; $2=$ yes $)$. Regarding alcohol use, participants were asked to rate the statement of "I drink too much alcohol or get drunk". Participants rating "very true or often true" were defined to have risky alcohol use. Based on the observations of the field investigators, none of the participants were under the influence of alcohol at the time of the study measurements.

Current Axis-I disorders were evaluated with Structured Interview for DSM-IV Axis I Disorders (SCID-I) (First et al., 1997). The presence of neurological disorders and times of having been unconscious were evaluated in the interview of neurological symptoms.

Level of functioning was measured with the Global Assessment of Functioning Scale (GAF) (Spitzer et al., 1996) in the SIPS interview. The validity and reliability of the GAF are demonstrated to be adequate (Sonesson et al., 2010; Startup et al., 2002). 
Full-scale intelligence quotient (FSIQ) was evaluated with the Vocabulary and Matrix Reasoning Scales of the WAIS-III (Wechsler Adult Intelligence Scale III, Finnish Edition) (Wechsler et al., 1997). The psychometric properties of the WAIS-III are shown to be excellent (Ryan and Ward, 1999). As has been done previously (Jukuri et al., 2013), we used the sum of the two scales as an indicator for full-scale intelligence quotient.

\subsection{Brain-imaging methods}

Resting-state BOLD (Blood Oxygen Level Dependent-signal) data were collected on a General Electric Signa $1.5 \mathrm{~T}$ whole-body system with an eight-channel receive coil, using an EPI (Echo Planar Imaging) GRE (Gradient Echo) sequence TR (Repetition Time) $1800 \mathrm{~ms}$, TE (Echo Time) $40 \mathrm{~ms}, 280$ time points, 28 oblique axial slices, slice thickness $4 \mathrm{~mm}$, inter-slice space $0.4 \mathrm{~mm}$, covering the whole brain, FOV (Field of View) $25.6 \mathrm{~cm} \times 25.6 \mathrm{~cm}$, with $64 \times 64$ matrix, parallel imaging factor 2 , and a flip angle of $90^{\circ}$. T1-weighted scans were imaged using a 3D FSPGR (Fast Spoiled Gradient echo) BRAVO (Brain Volume imaging) sequence (TR $12.1 \mathrm{~ms}$, TE $5.2 \mathrm{~ms}$, slice thickness $1.0 \mathrm{~mm}$, FOV $24.0 \mathrm{~cm}$, matrix $256 \times 256$, and flip angle $20^{\circ}$ ), and NEX (Number of Excitations) 1 in order to obtain anatomical images for co-registration of the fMRI data to standard space coordinates. Brain imaging methods were identical to those described in detail previously (Jukuri et al., 2013).

\subsection{Data preprocessing}

Neuroimaging data were analyzed with FSL (http://www.fmrib.ox. ac.uk/fsl, FSL 5.0.8) (Jenkinson and Smith, 2001, Jenkinson et al., 2002, Smith, 2002; Woolrich et al., 2001, 2004; Worsley, 2001) and AFNI (Cox, 1996). We conducted following steps in the pre-processing including brain extraction (AFNI's 3dSkullStrip), motion correction (MCFLIRT), linear co-registration (FLIRT), nonlinear normalization (FNIRT) to the $2 \mathrm{~mm}$ MNI-152 template, and detrending with AFNI's $3 \mathrm{dDetrend}$ due to the potential scanner-related effect on standard deviation. Relative and absolute root-mean-square (RMS) head displacement (millimeter) was determined from FSL's MCFLIRT and used as covariates in the model. FSL's FAST was used for the segmentation of T1-weighted structural images into white matter (WM), grey matter (GM) and cerebrospinal fluid (CSF).

For each study participant, the $\mathrm{CV}_{\mathrm{BOLD}}$ map was calculated as a ratio between the standard deviation of preprocessed BOLD-timeseries divided by the mean of preprocessed BOLD-timeseries in each voxel. This method has been used by (Kananen et al., 2018; Jahanian et al., 2014) and is similar to the method used by (Makedonov et al., 2013, 2016). We analyzed the association between SIPS and CV $_{\text {BOLD }}$ in the brain 1) globally by exploring the relationship between SIPS and the average $\mathrm{CV}_{\mathrm{BOLD}}$ in WM, GM and CSF and 2) locally by exploring the relationship between SIPS and $\mathrm{CV}_{\text {BOLD }}$ in a voxel-wise manner. We conducted the voxel-wise analysis using FSL's randomise tool (5000 permutations, applying demeaning and threshold free cluster enhancement (TFCE) (Smith and Nichols, 2009)). Sex, age, education, absolute and relative displacement were used as covariates in the above analyses.

\subsection{Statistical analyses}

The association of psychotic symptoms with $\mathrm{CV}_{\mathrm{BOLD}}$ in the brain was investigated using multivariable regression analyses (STATA MP, version 15.1). We predicted $C_{\text {BOLD }}$ in the brain by the SIPS score of positive symptoms. Separate models were conducted for $\mathrm{CV}_{\text {BOLD }}$ in CSF, WM, and GM. The analyses were controlled for age, sex, educational level, and absolute and relative displacement.
2.6. Comparison to meta-analytical maps of fMRI and VBM studies in schizophrenia patients

To compare our $\mathrm{CV}_{\mathrm{BOLD}}$ results with previous schizophrenia imaging studies, we conducted meta-analyses of the fMRI and VBM studies in patients with schizophrenia using the Brainmap database (search with Sleuth was conducted in August 2018). The aim was to explore the overlap of our findings with previously discovered results. We identified 50 fMRI studies and 27 VBM studies. We used both contrasts (i.e., schizophrenia >controls and schizophrenia<controls) in the metaanalysis of fMRI studies. This was due to the heterogeneity of the stimuli in these studies, and due to the fact that some of these studies used group $\mathrm{x}$ stimuli interaction. We analyzed only schizophrenia $<$ controls contrast in VBM studies as one of the most robust discoveries in schizophrenia has been lower in grey matter volume when compared to controls (Haijma et al., 2012). GingerALE (Eickhoff et al., 2009; Turkeltaub et al., 2002) with 1000 repetitions was used for the meta-analysis. The $p$-values for each meta-analysis were thresholded at a cluster level corrected threshold of $p<0.05$ (cluster-forming threshold at voxellevel $p<0.001$ ).

\section{Results}

Descriptive statistics of the study variables are shown in Table 1. There were altogether 142 participants with no psychotic symptoms (the SIPS score $=0$ ), 109 participants with mild psychotic-like symptoms (the SIPS score $=1-2$ ), 23 participants with prodromal symptoms of psychosis (the SIPS score $=3-5$ ), and 3 participants with psychotic symptoms (the SIPS score $=6$ ). Due to the low number of psychotic participants, the groups of psychotic symptoms and prodromal symptoms of psychosis were combined into one group (the SIPS score $=$ 3-6).

Table 2 shows the results of regression analyses when predicting $\mathrm{CV}_{\mathrm{BOLD}}$ in the brain by the SIPS scores. Participants in the study group with prodromal symptoms of psychosis or psychotic symptoms (the SIPS score $=3-6$ ) had higher $\mathrm{CV}_{\text {BOLD }}$ in CSF and WM when compared to participants with no psychotic symptoms $($ SIPS $=0)$. We did not obtain any consistent association between psychotic-like symptoms and the $\mathrm{CV}_{\mathrm{BOLD}}$ in GM. These findings are illustrated in Fig. 1a-c. All these findings were adjusted for age, sex, educational level, and absolute and relative parameters of displacement. The peak coordinates of the clusters that showed increased $\mathrm{CV}_{\text {BOLD }}$ in association with high SIPS scores are shown in Supplementary Table 1. We also rerun the analyses so that the participants with psychotic symptoms $(N=3)$ were excluded from the sample. The associations of the SIPS scores with $\mathrm{CV}_{\mathrm{BOLD}}$

Table 1

Means, standard deviations (SD), ranges, and frequencies of the study variables.

\begin{tabular}{|c|c|c|c|c|}
\hline & Mean & SD & Range & $\begin{array}{l}\text { Frequency } \\
(\%)\end{array}$ \\
\hline Age & 22.89 & 0.77 & $20.95-24.64$ & \\
\hline Sex (female) & & & & $148(53.4)$ \\
\hline SIPS positive symptoms & 0.90 & 1.25 & $0-6$ & \\
\hline No psychotic symptoms & & & & $142(51.3)$ \\
\hline Mild psychotic-like symptoms & & & & $109(39.4)$ \\
\hline Prodromal symptoms of psychosis & & & & $23(8.3)$ \\
\hline Psychotic symptoms & & & & $3(1.1)$ \\
\hline \multicolumn{5}{|l|}{ Educational level } \\
\hline Comprehensive school or less & & & & $119(43.0)$ \\
\hline Matriculation level & & & & $158(57.0)$ \\
\hline Smoker & & & & $132(47.7)$ \\
\hline Risky alcohol use & & & & $13(4.9)$ \\
\hline Current Axis-I disorder & & & & $51(18.4)$ \\
\hline Neurological disorder & & & & $18(6.5)$ \\
\hline $\begin{array}{l}\text { Previous times of unconsciousness } \\
\quad(>30 \mathrm{~min})\end{array}$ & & & & $3(1.1)$ \\
\hline Level of functioning & 80.12 & 11.61 & $21-96$ & \\
\hline Full-scale intelligence quotient & 96.93 & 25.12 & $25-160$ & \\
\hline
\end{tabular}


in CSF and WM remained significant (for further details, see Supplementary Table 2).

Additionally, we investigated whether the SIPS score correlated with motion parameters. The SIPS score did not correlate with the parameters of absolute motion ( $r=-0.023)$ or relative motion $(r=0.003)$. The plots about this non-significant correlation are available in Supplementary Fig. 1. This indicated that the association between SIPS and $\mathrm{CV}_{\mathrm{BOLD}}$ is not accounted for by differences in head motion during brain imaging between participants with different SIPS scores.

As additional analyses, we reran the multivariate regression analyses using the continuous variable of the current SIPS positive symptoms (ranging between 0 and 6). All the findings were replicated. Specifically, high score of SIPS positive symptoms was linked with higher $\mathrm{CV}_{\text {BOLD }}$ in CSF (beta $=0.142, p=0.001$ ) and higher $\mathrm{CV}_{\mathrm{BOLD}}$ in WM (beta $=0.116$, $p=0.012$ ), but not $\mathrm{CV}_{\mathrm{BOLD}}$ in $\mathrm{GM}$ (beta $=0.062, p=0.138$ ).

Voxel-wise analyses revealed that $\mathrm{CV}_{\mathrm{BOLD}}$ varied as a function of SIPS in different parts of WM and GM around the lateral ventricles in the current study. Specifically, SIPS correlated with $\mathrm{CV}_{\mathrm{BOLD}}$ in a bilateral way in the deep grey matter structures and white matter around the central CSF spaces. In the basal ganglia right side changes were dominant, while in the cranial white matter areas the left side showed more changes.

In the brain stem, there were two clusters in the pons. In particular, the periaqueductal grey matter was involved, and there was a continuous stream of voxel along the left brain peduncle. The right side of the thalamic nuclei was nearly totally covered with significant $\mathrm{CV}_{\mathrm{BOLD}}$ change; only the centerline half of the area 7 in FSL Thalamic parcellation was unaffected. On the left side, the changes were spatially smaller (also areas 1, 2, and 4 were spared). Bilaterally the posterior halves of the putamina and whole caudate nuclei were involved in $\mathrm{CV}_{\mathrm{BOLD}}$ change. Additionally, the right sides of both the amygdala and the hippocampi had $\mathrm{CV}_{\mathrm{BOLD}}$ change. Interestingly, on the left, the whole length of the hippocampi was affected, while in the right only the frontal third was affected.

In the cerebral cortical structures, the changes extended to primary sensorimotor cortices bilaterally and in the middle and frontal insula. Lateral parts of the paracingulate cortex were involved, but the changes were absent from the midline cingulate and other areas of the default mode network. Additionally, $\mathrm{CV}_{\mathrm{BOLD}}$ was affected in the right superior temporal gyrus. In the white matter, the most involved regions were the centrum semiovale areas around the CSF ventricles. Interestingly, the frontal parts of the white matter were involved, while the trigonal areas and posterior parts showed minimal involvement. In the cerebellum, the affected areas were 3,4, 7, 8 and 19 in MNI FSL, the largest cluster being posterior and above 4 th ventricle. Unthresholded $p$-value and T-stat maps are available in Supplementary Material.

Furthermore, we conducted meta-analyses of the previous fMRI and VBM studies among patients with schizophrenia to investigate the overlap between our findings and the previously discovered results. As shown in Fig. 2, these results overlapped only modestly with the meta-analyses of fMRI and VBM studies in patients with schizophrenia. The average map of the $\mathrm{CV}_{\mathrm{BOLD}}$ is provided in the Supplementary Fig. 2.

\section{Discussion}

For the first time, this study investigated the relationship of symptomatic psychosis risk with physiological fluctuation (as measured with $\mathrm{CV}_{\mathrm{BOLD}}$ ) in the brain. At the global level, participants in the study group with prodromal symptoms of psychosis or psychotic symptoms (the SIPS score = 3-6) had higher $\mathrm{CV}_{\text {BOLD }}$ in cerebrospinal fluid (CSF) and white matter (WM) but not in grey matter, when compared to participants with no psychotic symptoms (the SIPS score $=0$ ). Voxel-wise analyses revealed that $\mathrm{CV}_{\mathrm{BOLD}}$ was increased especially in periventricular white matter, basal ganglia, cerebellum and parts of the cortical structures. These findings were not explained by head motion. Additionally, we conducted meta-analyses of the previous fMRI and VBM studies among patients with schizophrenia to investigate the overlap between our findings and the previously discovered results. Those brain regions, which included alterations of physiological fluctuation in symptomatic psychosis risk, overlapped $<6 \%$ with the regions that were found to be affected in schizophrenia patients in the metaanalyses of previous fMRI and VBM studies. Hence, this implicates that our novel analyzing method of fMRI data (using the variation of BOLD-signal) might reveal new viewpoints to the neurofunctional alterations related to psychotic-like symptomatology.

The small amount of overlap between our results and the metaanalyses of fMRI and VBM data is expected, as $\mathrm{CV}_{\text {BOLD }}$ reflects a signal source that likely has not been measured in most previous fMRI and VBM studies. This signal is usually removed (e.g. via temporal filtering) and is not present in the T1-weighted data. Furthermore, most previous fMRI studies have limited their analyses to signals from grey matter, whereas the method of $\mathrm{CV}_{\mathrm{BOLD}}$ enabled us to investigate physiological fluctuation also in white matter and cerebrospinal fluid in fMRI data. The $\mathrm{CV}_{\mathrm{BOLD}}$ findings, thus, produce new information that has been out of reach of previous fMRI and VBM studies.

Previously, patients with epilepsy, Alzheimer's disease, and acute ischemic stroke are found to have alterations in the physiological noise of the brain (Makedonov et al., 2013, 2016; Kananen et al., 2018; Khalil et al., 2017; Wang et al., 2008). Moreover, schizophrenia is found to be related to increased physiological noise in the white matter (Cheng et al., 2015). This study was the first to demonstrate that prodromal syndromes are associated with increased $\mathrm{CV}_{\mathrm{BOLD}}$ in white matter, especially around the third ventricle and lateral ventricles. Previously, this would have been considered to be a factor to be recognized and removed while focusing on changes in neuro-hemodynamic responses or fluctuations. However, as indicated by the latest intra-vital microscopy results, hypertension-induced abnormal pulsations like reduce brain waste removal and, therefore, abnormal pulsations themselves can be a more direct measure of the underlying pathology (Mestre et al., 2018; Kiviniemi et al., 2016). The current results add proof to the idea that physiological processes maintaining interstitial brain homeostasis may be altered also in psychosis. Further, recent evidence suggests that the glymphatic paravascular cerebrospinal fluid brain clearance may be altered in normal pressure hydrocephalus (Eide and Sorteberg, 2016; Ringstad et al., 2017) that is linked to schizophrenia (Vanhala et al., 2018).

Our finding about the link between psychotic-like symptoms and increased $\mathrm{CV}_{\text {BOLD }}$ in CSF is in accord with previous findings. For example, there is evidence that patients with schizophrenia have enlarged ventricles (Shenton et al., 2001; Steen et al., 2006) and, conversely, normal pressure hydrocephalus is linked to schizophrenia (Vanhala et al., 2018) and alterations in glymphatic paravascular cerebrospinal fluid brain clearance (Eide and Sorteberg, 2016; Ringstad et al., 2017). Moreover, psychotic patients are found to have abnormal neurochemical composition of cerebrospinal fluid, for example, increased concentrations of some cytokines (Nikkilä et al., 2001; Schwieler et al., 2015), bioactive lipids (Koethe et al., 2009), proteins and peptides in cerebrospinal fluid (Huang et al., 2006; Thompson et al., 2003). The abnormal composition of cerebrospinal fluid, in turn, is known to affect the secretion and flow of cerebrospinal fluid (Sakka et al., 2011). Additionally, as indicated by the latest intra-vital microscopy results, abnormal blood pulsations may likely reduce brain waste removal (Mestre et al., 2018; Kiviniemi et al., 2016) and increased level of brain waste, in turn, may potentially increase "noise fluctuation" in the brain. Taken together, among individuals with psychotic-like symptoms, increased $\mathrm{CV}_{\mathrm{BOLD}}$ in cerebrospinal fluid may partly derive from an altered amount and neurochemical composition of cerebrospinal fluid and impairments in the glymphatic system.

At the global (whole-brain) level, psychotic-like symptoms were not associated with $\mathrm{CV}_{\mathrm{BOLD}}$ in grey matter. This may be related to the findings that the most marked cardiovascular pulsations occur in the CSF areas near the major vessels (Kiviniemi et al., 2016; Raitamaa et al., 
(a)



(b)

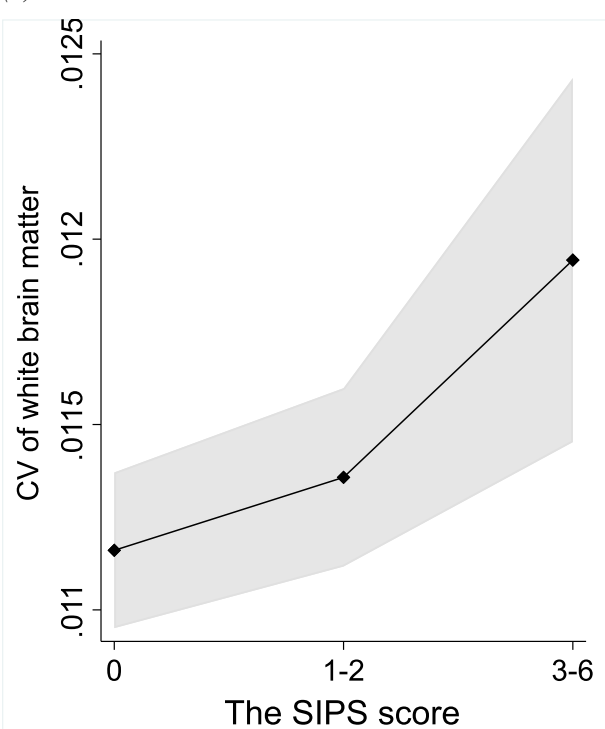

(c)

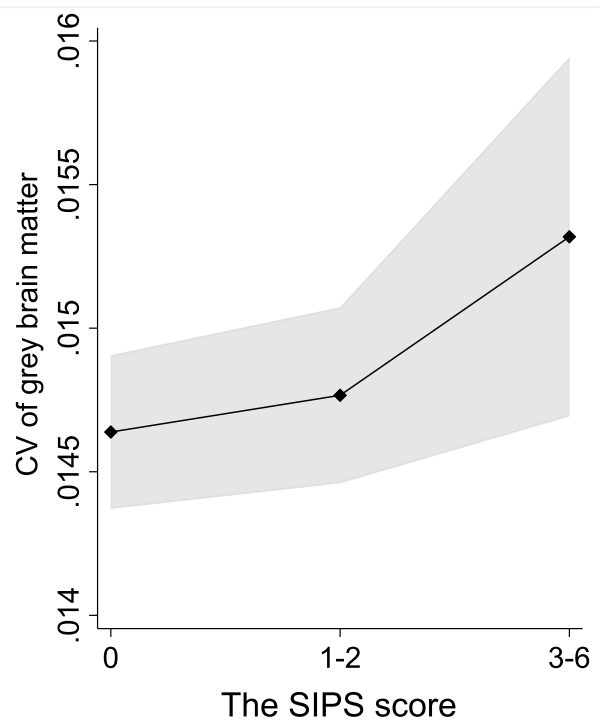

2018). Importantly, however, our voxel-wise analyses showed that psychotic-like symptoms correlated with increased $\mathrm{CV}_{\text {BOLD }}$ in the deep grey matter structures around the central CSF spaces (i.e. basal ganglia, amygdala, thalamic nuclei, hippocampus, cerebellum). Previous studies in schizophrenia patients have shown that positive symptoms correlate with increased activity levels in the striatum (Sorg et al., 2012). Further, schizophrenia-related cognitive disturbances are linked to the altered activity levels in the cerebellum (Lungu et al., 2012). Importantly, our findings provided evidence that psychotic-like symptoms are related to the increased fluctuation of the BOLD-signal in the same brain regions. Hence, our findings tentatively arise the interesting question whether some of the previously found associations between psychotic symptoms and fMRI activity might partly reflect increased variation of the BOLDsignal.

Increased $\mathrm{CV}_{\mathrm{BOLD}}$ in the brain may also reflect psychosis-related changes in cardiovascular and respiratory activities. Specifically, psychotic disorders have been related to reduced baroreflex sensitivity and lower reactivity of heart-rate variability to the demands of the task (Bär et al., 2007a; Valkonen-Korhonen et al., 2003), reduced parasympathetic activity (i.e. vagal activity) (Mujica-Parodi et al., 2005), increased QT variability (Bär et al., 2007b), and peripheral endothelial dysfunction (Israel et al., 2011). Respiratory waves and cardiac pulsation, in turn, are found to affect the flow of cerebrospinal fluid in the ventricles and surrounding tissues (Dagli et al., 1999; Sakka et al., 2011). Consequently, psychosis seems to be related to an imbalance between sympathetic and parasympathetic systems and other alterations in cardiophysiological activities that may increase the unstability of the neurophysiological systems and, thus, the physiological fluctuation in the brain.

This study had some methodological limitations. Firstly, the group of participants with psychosis-level symptoms consisted of only three individuals. Hence, our findings cannot be directly generalized to patients with psychotic disorders. However, the link between schizophrenia and increased physiological noise in the brain has been demonstrated previously (Cheng et al., 2015). Hence, the primary aim of this study was to investigate individuals at symptomatic risk for developing psychosis, in line with the recent continuum-based viewpoint on early stages of psychosis (McGorry et al., 2007; Van Os et al., 2009). Additionally, our aim was to introduce a novel method for analyzing fMRI data in order to obtain previously uninvestigated sources of signal fluctuation. Future studies are needed to replicate our findings in larger samples.

Our sample was comparatively heterogeneous, including participants with familial risk for psychosis, attention-deficit hyperactivity disorder, or current Axis-I disorder. Previously, the high comorbidity of psychotic symptoms with a wide variety of other symptoms has been widely demonstrated (Fusar-Poli et al., 2012; Gaitatzis et al., 2004; Karatekin et al., 2010; Keshavan et al., 2003; Lee and Weintraub, 2012; Schuckit, 2006). Hence, individuals with psychotic symptomatology are known to represent a highly heterogeneous population. Consequently, our heterogeneous sample may enhance the generalizability of the findings to various populations.

In this study, brain scanning was conducted using the General Electric Signa 1.5 T fMRI system, which can be nowadays considered relatively slow TR (sampling time $1.8 \mathrm{~s}$ ) and large voxel size (see Jukuri et al., 2013). It is relatively rarely used in schizophrenia research. On the other hand, it likely enabled us to obtain a relatively large amount of physiological fluctuation in the BOLD signal. Hence, our brain imaging data likely provided exceptional possibilities for investigating $\mathrm{CV}_{\mathrm{BOLD}}$ in the brain.

This study had also several substantial strengths. Firstly, this was the first study to investigate whether symptomatic psychosis risk might be

Fig. 1. a-c. Predicted marginal means with $95 \%$ confidence intervals of CV in cerebrospinal fluid (a), white brain matter (b), and grey brain matter (c) for participants with different levels of SIPS positive symptoms. Adjusted for age, sex, educational level, and absolute and relative displacement. 
Table 2

Results of linear regression analyses, when predicting $\mathrm{CV}_{\mathrm{BOLD}}$ in cerebrospinal fluid (CSF), white brain matter, and grey brain matter by SIPS positive symptoms.

\begin{tabular}{|c|c|c|c|c|c|c|c|c|c|}
\hline & \multicolumn{3}{|c|}{ Model 1: $\mathrm{CV}_{\mathrm{BOLD}}$ in CSF } & \multicolumn{3}{|c|}{ Model 2: $\mathrm{CV}_{\mathrm{BOLD}}$ in white matter } & \multicolumn{3}{|c|}{ Model 3: $\mathrm{CV}_{\mathrm{BOLD}}$ in grey matter } \\
\hline & Beta & B & $p$ & Beta & B & $p$ & Beta & B & $p$ \\
\hline Age & -0.199 & -0.001 & $<0.001$ & -0.136 & 0.000 & 0.003 & -0.096 & 0.000 & 0.023 \\
\hline $\operatorname{Sex}^{\mathrm{a}}$ & -0.342 & -0.002 & $<0.001$ & -0.350 & -0.001 & $<0.001$ & -0.237 & -0.001 & $<0.001$ \\
\hline Educational level & 0.045 & 0.000 & 0.315 & -0.033 & 0.000 & 0.469 & 0.017 & 0.000 & 0.684 \\
\hline Absolute displacement & -0.178 & -0.002 & $<0.001$ & -0.226 & -0.001 & $<0.001$ & -0.180 & -0.001 & $<0.001$ \\
\hline Relative displacement & 0.587 & 0.077 & $<0.001$ & 0.555 & 0.038 & $<0.001$ & 0.707 & 0.067 & $<0.001$ \\
\hline \multicolumn{10}{|l|}{ SIPS positive symptoms } \\
\hline No psychotic symptoms ${ }^{\mathrm{b}}$ & - & - & - & & - & - & - & - & - \\
\hline Mild psychotic-like symptoms & 0.052 & 0.000 & 0.253 & 0.057 & 0.000 & 0.228 & 0.027 & 0.000 & 0.538 \\
\hline Prodromal symptoms of psychosis or psychotic symptoms & 0.146 & 0.002 & 0.001 & 0.136 & 0.001 & 0.004 & 0.085 & 0.001 & 0.051 \\
\hline
\end{tabular}

a Male as the reference group.

b The reference group. $N=276$.

linked with $\mathrm{CV}_{\mathrm{BOLD}}$ in the brain. Hence, we used a novel method for investigating the signal fluctuation in fMRI data. Secondly, to provide an interpretation of the findings, we also compared the findings with meta-analyses of the previous fMRI and VBM studies among patients with schizophrenia. This provided valuable information about how the novel method of $\mathrm{CV}_{\mathrm{BOLD}}$ might enable identifying such alterations in white matter and cerebrospinal fluid that have not been identified with the traditional analytical methods in fMRI data. Thirdly, the NFBC 1986 birth cohort provided a sample of roughly same-age participants in their young adulthood, i.e. the age phase with highest risk for psychosis (Beiser et al., 1993). Furthermore, the sample was comparatively large $(N>270)$ that increased the statistical power and provided possibilities to obtain associations between psychosis risk and physiological fluctuation in the brain. Finally, symptomatic psychosis risk was evaluated with a widely used structural interview (SIPS) that is demonstrated to be a reliable measure of prodromal syndromes (Miller et al., 2003).

In conclusion, at the global level, psychotic-like symptoms were linked to higher $\mathrm{CV}_{\mathrm{BOLD}}$ in cerebrospinal fluid (CSF) and white matter (WM). Voxel-wise analyses showed that in individuals with psychotic-like symptoms, $\mathrm{CV}_{\text {BOLD }}$ was increased especially in periventricular white matter, basal ganglia, cerebellum and parts of the cortical structures. The overlap between these results and the meta-analyses of previous fMRI and VBM data was small, suggesting that $\mathrm{CV}_{\mathrm{BOLD}}$ might reflect such a signal source that has not been investigated in previous fMRI and VBM studies. Future studies with larger study samples should investigate whether our results can be generalized to other populations. a)
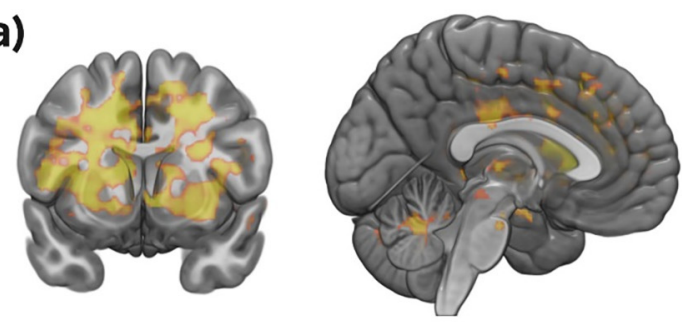

b)

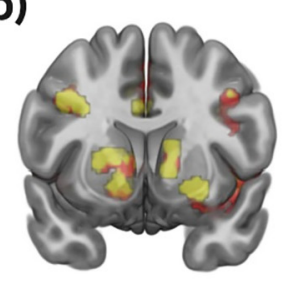

c)

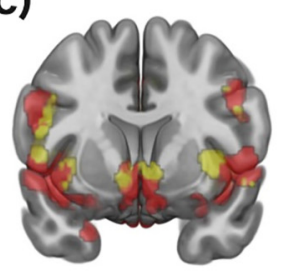

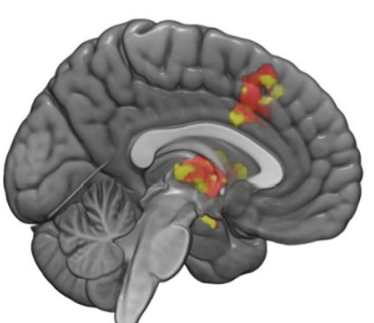

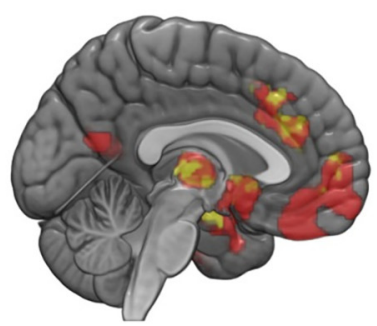

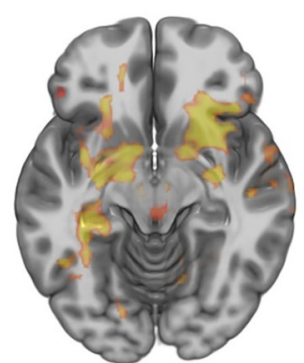

Main effect of SIPS
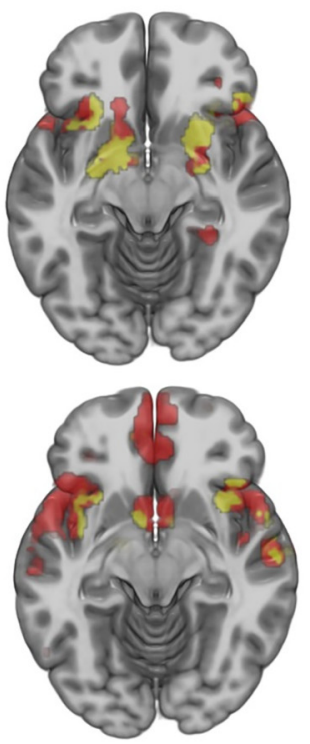

Overlap with SZ fMRI

SZ fMRI

\section{Overlap with SZ VBM}

SZ VBM

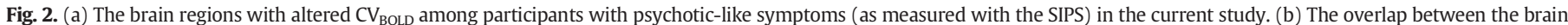

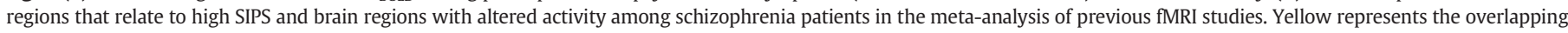

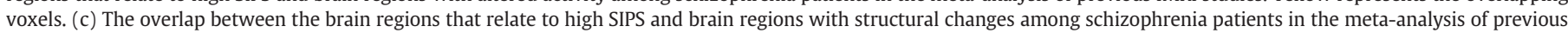
VBM studies. Yellow represents the overlapping voxels. (For interpretation of the references to colour in this figure legend, the reader is referred to the web version of this article.) 
Supplementary data to this article can be found online at https://doi. org/10.1016/j.schres.2019.11.029.

\section{Contributors}

A.S. drafted the manuscript and conducted statistical analyses. J.L. conducted data preprocessing, assisted with statistical analyses, and contributed to interpretation of the results and writing of the manuscript. V.K. assisted with statistical analyses and contributed to interpretation of the results and writing of the manuscript. J.H., T.T., M.H and J.V. contributed to the interpretation of the results and collaborated with writing the manuscript. J.V. contributed to the data collection. All authors contributed to and have approved the final manuscript.

\section{Declaration of competing interest}

The authors declare that they have no conflict of interest.

\section{Acknowledgments}

This study was supported financially by the Academy of Finland (J.V., grant number 124257, 141042, 212818, 214273, and 308555; V.K., Terva grant), the Sigrid Juselius Foundation (J.V.), the Gyllenberg Foundation (J.V.), the Medical Research Council (MRC) (V.K.), the Jane and Aatos Erkko Foundation (V.K.), the Alfred Kordelin Foundation (J.L.), the Orion Research Foundation (J.L., T.T.), the Yrjö Jahnsson Foundation (JL), Jalmari and Rauha Ahokas Foundation (JL), and the Finnish Medical Foundation (J.L.T.T.). The NFBC study has been financially supported by EU QLG1-CT-2000-01643 (EUROBLCS) grant number E51560, NorFA grant number 731, 20056, 30167, USA/NIHH 2000 G DF682 grant number 50945. We thank all the cohort members and researchers who have participated in the study. We also wish to acknowledge the work of the NFBC project center.

\section{Role of the funding source}

The funding source had no role in study design, data collection, data analysis, data interpretation, writing of the report, or in the decision to submit the article for publication.

\section{References}

Bär, K.J., Boettger, M.K., Berger, S., et al., 2007a. Decreased baroreflex sensitivity in acute schizophrenia. J. Appl. Physiol. 102, 1051-1056. https://doi.org/10.1152/ japplphysiol.00811.2006.

Bär, K.J., Koschke, M., Boettger, M.K., et al., 2007b. Acute psychosis leads to increased QT variability in patients suffering from schizophrenia. Schizophr. Res. 95, 115-123. https://doi.org/10.1016/j.schres.2007.05.034.

Beiser, M., Erickson, D., Fleming, J.A., Iacono, W.G., 1993. Establishing the onset of psychotic illness. Am. J. Psychiatr. 150, 1349-1354.

Birn, R.M., 2012. The role of physiological noise in resting-state functional connectivity. Neuroimage 62, 864-870. https://doi.org/10.1016/j.neuroimage.2012.01.016.

Birn, R.M., Diamond, J.B., Smith, M.A., Bandettini, P.A., 2006. Separating respiratoryvariation-related fluctuations from neuronal-activity-related fluctuations in fMR Neuroimage 31, 1536-1548. https://doi.org/10.1016/j.neuroimage.2006.02.048.

Cheng, H., Newman, S.D., Kent, J.S., et al., 2015. White matter abnormalities of microstructure and physiological noise in schizophrenia. Brain Imaging and Behavior 9 868-877. https://doi.org/10.1007/s11682-014-9349-1.

Cox, R.W., 1996. AFNI: software for analysis and visualization of functional magnetic resonance neuroimages. Comput. Biomed. Res. 29, 162-173.

Dagli, M.S., Ingeholm, J.E., Haxby, J.V., 1999. Localization of cardiac-induced signal change in fMRI. Neuroimage 9, 407-415. https://doi.org/10.1006/nimg.1998.0424.

Dreha-Kulaczewski, S., Joseph, A.A., Merboldt, K.D., Ludwig, H.C., Gärtner, J., Frahm, J. 2015. Inspiration is the major regulator of human CSF flow. J. Neurosci. 35, 2485-2491. https://doi.org/10.1523/JNEUROSCI.3246-14.2015.

Eickhoff, S.B., Laird, A.R., Grefkes, C., Wang, L.E., Zilles, K., Fox, P.T., 2009. Coordinate-based activation likelihood estimation meta-analysis of neuroimaging data: a randomeffects approach based on empirical estimates of spatial uncertainty. Hum. Brain Mapp. 30, 2907-2926. https://doi.org/10.1002/hbm.20718.

Eide, P.K., Sorteberg, W., 2016. Outcome of surgery for idiopathic normal pressure hydrocephalus: role of preoperative static and pulsatile intracranial pressure. World Neurosurgery 86, 186-193. https://doi.org/10.1016/j.wneu.2015.09.067.

First, M.B., Spitzer, R.L., Gibbon, M., Williams, J.B., 1997. Structured Clinical Interview for DSM-IV-TR Axis I Disorders, Research Version. Patient edition. SCID-I/P https://doi. org/10.1093/schbul/sbs136.

Fusar-Poli, P., Nelson, B., Valmaggia, L., Yung, A.R., McGuire, P.K., 2012. Comorbid depressive and anxiety disorders in 509 individuals with an at-risk mental state: impact on psychopathology and transition to psychosis. Schizophr. Bull. 40, 120-131. https:// doi.org/10.1093/schbul/sbs136.

Gaitatzis, A., Trimble, M.R., Sander, J.W., 2004. The psychiatric comorbidity of epilepsy. Acta Neurol. Scand. 110, 207-220.

Haijma, S.V., Van Haren, N., Cahn, W., Koolschijn, P.C.M., Hulshoff Pol, H.E., Kahn, R.S. 2012. Brain volumes in schizophrenia: a meta-analysis in over 18000 subjects Schizophr. Bull. 39, 1129-1138. https://doi.org/10.1093/schbul/sbs118.

Huang, J.T.J., Leweke, F.M., Oxley, D., et al., 2006. Disease biomarkers in cerebrospinal fluid of patients with first-onset psychosis. PLoS Med. 3, e428. https://doi.org/10.1371/ journal.pmed.0030428.
Israel, A.K., Seeck, A., Boettger, M.K., et al., 2011. Peripheral endothelial dysfunction in patients suffering from acute schizophrenia: a potential marker for cardiovascular morbidity. Schizophr. Res. 128, 44-50. https://doi.org/10.1016/j.schres.2011.02.007.

Jahanian, H., Ni, W.W., Christen, T., Moseley, M.E., Tamura, M.K., Zaharchuk, G., 2014. Spontaneous BOLD signal fluctuations in young healthy subjects and elderly patients with chronic kidney disease. PLoS One 9, e92539. https://doi.org/10.1371/journal. pone.0092539.

Järvelin, M.R., Elliott, P., Kleinschmidt, I., et al., 1997. Ecological and individual predictors of birthweight in a northern Finland birth cohort 1986. Paediatr. Perinat. Epidemiol. $11,298-312$

Jenkinson, M., Smith, S., 2001. A global optimisation method for robust affine registration of brain images. Med. Image Anal. 5, 143-156.

Jenkinson, M., Bannister, P., Brady, M., Smith, S., 2002. Improved optimization for the robust and accurate linear registration and motion correction of brain images. Neuroimage 17, 825-841.

Jukuri, T., Kiviniemi, V., Nikkinen, J., et al., 2013. Default mode network in young people with familial risk for psychosis - the Oulu Brain and Mind study. Schizophr. Res. 143, 239-245. https://doi.org/10.1016/j.schres.2012.11.020.

Kananen, J., Tuovinen, T., Ansakorpi, H., et al., 2018. Altered physiological brain variation in drug-resistant epilepsy. Brain and Behavior 8, e01090. https://doi.org/10.1002/ brb3.1090.

Karatekin, C., White, T., Bingham, C., 2010. Shared and nonshared symptoms in youthonset psychosis and ADHD. J. Atten. Disord. 14, 121-131. https://doi.org/10.1177/ 1087054709347434

Keshavan, M.S., Sujata, M., Mehra, A., Montrose, D.M., Sweeney, J.A., 2003. Psychosis proneness and ADHD in young relatives of schizophrenia patients. Schizophr. Res. 59, 85-92.

Khalil, A.A., Ostwaldt, A.C., Nierhaus, T., et al., 2017. Relationship between changes in the temporal dynamics of the blood-oxygen-level-dependent signal and hypoperfusion in acute ischemic stroke. Stroke 48, 925-931. https://doi.org/10.1161/ STROKEAHA.116.015566/-/DC1.

Kiviniemi, V., Wang, X., Korhonen, V., et al., 2016. Ultra-fast magnetic resonance encephalography of physiological brain activity-glymphatic pulsation mechanisms. J. Cereb. Blood Flow Metab. 36, 1033-1045. https://doi.org/10.1177/0271678X15622047.

Koethe, D., Giuffrida, A., Schreiber, D., et al., 2009. Anandamide elevation in cerebrospinal fluid in initial prodromal states of psychosis. Br. J. Psychiatry 194, 371-372. https:// doi.org/10.1192/bjp.bp.108.053843.

Krüger, G., Glover, G.H., 2001. Physiological noise in oxygenation-sensitive magnetic resonance imaging. Magnetic Resonance in Medicine: An Official Journal of the International Society for Magnetic Resonance in Medicine 46, 631-637.

Lee, A.H., Weintraub, D., 2012. Psychosis in Parkinson's disease without dementia: common and comorbid with other non-motor symptoms. Mov. Disord. 27, 858-863. https://doi.org/10.1002/mds.25003.

Lungu, O., Barakat, M., Laventure, S., Debas, K, Proulx, S. Luck, D., Stip, E., 2012. The incidence and nature of cerebellar findings in schizophrenia: a quantitative review of fMRI literature. Schizophr. Bull. 39, 797-806. https://doi.org/10.1093/schbul/sbr193.

Makedonov, I., Black, S.E., MacIntosh, B.J., 2013. BOLD fMRI in the white matter as a marker of aging and small vessel disease. PLoS One 8, e67652. https://doi.org/ 10.1371/journal.pone.0067652.

Makedonov, I., Chen, J.J., Masellis, M., MacIntosh, B.J., 2016. Alzheimer's disease neuroimaging initiative. Physiological fluctuations in white matter are increased in Alzheimer's disease and correlate with neuroimaging and cognitive biomarkers. Neurobiol. Aging 37, 12-18. https://doi.org/10.1016/j.neurobiolaging.2015.09.010.

McGlashan, T.H., Miller, T.J., Woods, S.W., Hoffman, R.E., Davidson, L., 2001. Instrument for the assessment of prodromal symptoms and states. Early Intervention in Psychotic Disorders. Springer, Dordrecht, pp. 135-149.

McGorry, P.D., Killackey, E., Yung, A.R., 2007. Early intervention in psychotic disorders: detection and treatment of the first episode and the critical early stages. Med. J. Aust. 187 (S8).

Mestre, H., Tithof, J., Du, T., Song, W., Peng, W., Sweeney, A.M., ... Kelley, D.H., 2018. Flow of cerebrospinal fluid is driven by arterial pulsations and is reduced in hypertension. Nature Communications 9, 4878 .

Miller, T.J., McGlashan, T.H., Rosen, J.L., et al., 2003. Prodromal assessment with the structured interview for prodromal syndromes and the scale of prodromal symptoms: predictive validity, interrater reliability, and training to reliability. Schizophr. Bull. 29, 703-715.

Mujica-Parodi, L.R., Yeragani, V., Malaspina, D., 2005. Nonlinear complexity and spectral analyses of heart rate variability in medicated and unmedicated patients with schizophrenia. Neuropsychobiology 51, 10-15. https://doi.org/10.1159/000082850.

Murphy, K., Birn, R.M., Handwerker, D.A., Jones, T.B., Bandettini, P.A., 2009. The impact of global signal regression on resting state correlations: are anti-correlated networks introduced. Neuroimage 44, 893-905. https://doi.org/10.1016/j.neuroimage.2008.09.036.

Nikkilä, H.V., Müller, K., Ahokas, A., Rimón, R., Andersson, L.C., 2001. Increased frequency of activated lymphocytes in the cerebrospinal fluid of patients with acute schizophrenia. Schizophr. Res. 49, 99-105.

Ogawa, S., Lee, T.M., Kay, A.R. Tank, D.W., 1990. Brain magnetic resonance imaging with contrast dependent on blood oxygenation. Proc. Natl. Acad. Sci. 87, 9868-9872. https://doi.org/10.1073/pnas.87.24.9868.

Raitamaa, L. Korhonen, V., Huotari, N., et al, 2018. Breath hold effect on cardiovascular brain pulsations-a multimodal magnetic resonance encephalography study. J. Cereb. Blood Flow Metab. https://doi.org/10.1177/0271678X18798441 $0271678 \times 18798441$.

Ringstad, G., Vatnehol, S.A.S., Eide, P.K., 2017. Glymphatic MRI in idiopathic normal pressure hydrocephalus. Brain 140, 2691-2705. https://doi.org/10.1093/brain/awx191.

Ryan, J.J., Ward, L.C., 1999. Validity, reliability, and standard errors of measurement for two seven-subtest short forms of the Wechsler Adult Intelligence Scale-III. Psychol. Assess. 11, 207-211. 
Sakka, L., Coll, G., Chazal, J., 2011. Anatomy and physiology of cerebrospinal fluid. Eur. Ann. Otorhinolaryngol. Head Neck Dis. 128, 309-316. https://doi.org/10.1016/j. anorl.2011.03.002.

Schuckit, M.A., 2006. Comorbidity between substance use disorders and psychiatric conditions. Addiction 101, 76-88. https://doi.org/10.1111/j.1360-0443.2006.01592.x.

Schwieler, L., Larsson, M.K., Skogh, E., et al., 2015. Increased levels of IL-6 in the cerebrospinal fluid of patients with chronic schizophrenia-significance for activation of the kynurenine pathway. J. Psychiatry Neurosci. 40, 126.

Shenton, M.E., Dickey, C.C., Frumin, M., McCarley, R.W., 2001. A review of MRI findings in schizophrenia. Schizophr. Res. 49, 1-52. https://doi.org/10.1016/S0920-9964(01) 00163-3.

Smith, S.M., 2002. Fast robust automated brain extraction. Hum. Brain Mapp. 17, 143-155.

Smith, S.M., Nichols, T.E., 2009. Threshold-free cluster enhancement: addressing problems of smoothing, threshold dependence and localisation in cluster inference. Neuroimage 44, 83-98. https://doi.org/10.1016/j.neuroimage.2008.03.061.

Sonesson, O., Tjus, T., Arvidsson, H., 2010. Reliability of a functioning scale (GAF) among psychiatric ward staff. Nordic Psychology 62, 53-64. https://doi.org/10.1027/19012276/a000005.

Sorg, C., Manoliu, A., Neufang, S., Myers, N., Peters, H., Schwerthöffer, D., Förstl, H., 2012. Increased intrinsic brain activity in the striatum reflects symptom dimensions in schizophrenia. Schizophr. Bull. 39, 387-395. https://doi.org/10.1093/schbul/sbr184.

Spitzer, R.L., Gibbon, M., Williams, J.B.W., Endicott, J., 1996. Global assessment of functioning (GAF) scale. Outcome Assessment in Clinical Practice, pp. 76-78.

Startup, M., Jackson, M.C., Bendix, S., 2002. The concurrent validity of the Global Assessment of Functioning (GAF). Br. J. Clin. Psychol. 41, 417-422.

Steen, R.G., Mull, C., Mcclure, R., Hamer, R.M., Lieberman, J.A., 2006. Brain volume in firstepisode schizophrenia: systematic review and meta-analysis of magnetic resonance imaging studies. Br. J. Psychiatry 188, 510-518. https://doi.org/10.1192/bjp.188.6.510.

Thompson, P.M., Kelley, M., Yao, J., Tsai, G., van Kammen, D.P., 2003. Elevated cerebrospinal fluid SNAP-25 in schizophrenia. Biol. Psychiatry 53, 1132-1137.

Tuovinen, T., Rytty, R., Moilanen, V., et al., 2017. The effect of gray matter ICA and coefficient of variation mapping of BOLD data on the detection of functional connectivity changes in Alzheimer's disease and bvFTD. Front. Hum. Neurosci. 10, 680. https:// doi.org/10.3389/fnhum.2016.00680.
Turkeltaub, P.E., Eden, G.F., Jones, K.M., Zeffiro, T.A., 2002. Meta-analysis of the functional neuroanatomy of single-word reading: method and validation. Neuroimage 16 765-780.

Valkonen-Korhonen, M., Tarvainen, M.P., Ranta-Aho, P., et al., 2003. Heart rate variability in acute psychosis. Psychophysiology 40, 716-726.

Van Os, J., Linscott, R.J., Myin-Germeys, I., Delespaul, P., Krabbendam, L., 2009. A systematic review and meta-analysis of the psychosis continuum: evidence for a psychosis proneness-persistence-impairment model of psychotic disorder. Psychol. Med. 39, 179-195. https://doi.org/10.1017/S0033291708003814.

Vanhala, V., Junkkari, A., Korhonen, V.E., et al., 2018. Prevalence of schizophrenia in idiopathic Normal pressure hydrocephalus. Neurosurgery, nyy147 https://oi.org/ 10.1093/neuros/nyy147.

Veijola, J., Mäki, P., Jääskeläinen, E., et al., 2013. Young people at risk for psychosis: case finding and sample characteristics of the Oulu Brain and Mind Study. Early Intervention in Psychiatry 7, 146-154. https://doi.org/10.1111/j.1751-7893.2012.00360.x.

Wang, H.H., Menezes, N.M., Zhu, M.W., et al., 2008. Physiological noise in MR images: an indicator of the tissue response to ischemia. J. Magn. Reson. Imaging 27, 866-871. https://doi.org/10.1002/jmri.21007.

Wechsler, D., Coalson, D.L., Raiford, S.E., 1997. WAIS-III: Wechsler Adult Intelligence Scale. Psychological Corporation, San Antonio, TX.

Weissenbacher, A., Kasess, C., Gerstl, F., Lanzenberger, R., Moser, E., Windischberger, C., 2009. Correlations and anticorrelations in resting-state functional connectivity MRI: a quantitative comparison of preprocessing strategies. Neuroimage 47, 1408-1416.

Wise, R.G., Ide, K., Poulin, M.J., Tracey, I., 2004. Resting fluctuations in arterial carbon dioxide induce significant low frequency variations in BOLD signal. Neuroimage 21 1652-1664. https://doi.org/10.1016/j.neuroimage.2003.11.025.

Woolrich, M.W., Ripley, B.D., Brady, M., Smith, S.M., 2001. Temporal autocorrelation in univariate linear modeling of FMRI data. Neuroimage 14, 1370-1386. https://doi org/10.1006/nimg.2001.0931.

Woolrich, M.W., Behrens, T.E., Beckmann, C.F., Jenkinson, M., Smith, S.M., 2004. Multilevel linear modelling for FMRI group analysis using Bayesian inference. Neuroimage 21, 1732-1747. https://doi.org/10.1016/j.neuroimage.2003.12.023.

Worsley, K.J., 2001. Statistical analysis of activation images. Functional MRI: An Introduction to Methods 14, 251-270. 